\title{
Escala de aprendizaje autogestivo en estudiantes universitarios de la carrera de Psicología de un sistema en línea
}

\section{Scale of self-management learning in university students of the career of Psychology of an online system}

\author{
Consuelo Rubi Rosales Piña ${ }^{1}$ \\ https://orcid.org/0000-0002-0605-1859 \\ Ricardo Sánchez Medina ${ }^{2}$ \\ https://orcid.org/0000-0003-4268-3025 \\ Sandra Ivonne Muñoz Maldonado ${ }^{3}$ \\ https://orcid.org/0000-0002-9392-5023 \\ Universidad Nacional Autónoma de México. Facultad de Estudios Superiores Iztacala
}

Recibido: 02-05-2019

Aceptado: 01-08-2019

\section{Cita Recomendada}

Rosales, C., Sánchez, R. \& Muñoz, S. (2019). Escala de aprendizaje autogestivo en estudiantes universitarios de la carrera de psicología de un sistema en línea. Hamut'ay, 6(2), 102-125. http://dx.doi.org/10.21503/hamu.v6i2.1778

\section{RESUMEN}

El objetivo de la presente investigación fue diseñar y validar una escala de aprendizaje autogestivo en estudiantes universitarios de un sistema en línea. Para ello se utilizó un diseño instrumental dividido en dos fases, en la primera fase se evalúo a 149 estudiantes universitarios de la modalidad en línea, a través de la estrategia de redes semánticas naturales modificadas, para conocer el significado psicológico de los estímulos presentados, las palabras definidoras más recurrentes fueron: organización, autodidacta, compromiso, satisfacción, responsabilidad; que sirvieron como base para diseñar la propuesta de un instrumento con el propósito de evaluar el aprendizaje autogestivo en una escala tipo Likert con cinco opciones de respuesta. En la segunda fase, participaron 929 estudiantes universitarios de un sistema en línea, a quienes se aplicó la propuesta y se obtuvieron las propiedades psicométricas de la escala. Se realizó un análisis factorial exploratorio con rotación varimax, que dio como resultado seis factores (afecto negativo para el aprendizaje en línea, aptitud para el aprendizaje autogestivo, responsabilidad en el proceso de aprendizaje, acciones para aprender de manera independiente, recursos tecnológicos para el aprendizaje y recursos personales para el aprendizaje), que explican el 59.74\% de la varianza, y una confiabilidad de .846. Los resultados se discuten en términos de la importancia de replicar este instrumento a muestras

\footnotetext{
${ }^{1}$ Profesora de Asignatura A definitiva adscrita a la carrera de Psicología del SUAyED de la Facultad de Estudios Superiores Iztacala, UNAM. Su línea de investigación es el estudio de los procesos familiares y de pareja y la creación de cursos en línea autogestivos. Miembro del grupo de Investigación en Procesos Psicológicos y Sociales (GIPPS). E-mail: rubi.rosales@iztacala.unam.mx

${ }^{2}$ Profesor de Carrera Titular A definitivo adscrito a la carrera de Psicología del SUAyED - UNAM. Su línea de investigación es en salud sexual en contextos vulnerables y en la creación de cursos en línea autogestivos. Miembro del grupo de Investigación GIPPS y responsable del Laboratorio de Psicología, Tecnología y Salud. E-mail: ricardo.sanchez@iztacala.unam.mx

${ }^{3}$ Profesora de Carrera Asociada C adscrita a la carrera de Psicología del SUAyED - UNAM. Su línea de investigación es en tecnologías aplicadas a la educación e intervención y la creación de cursos en línea autogestivos. Miembro del grupo de Investigación GIPPS. E-mail: sandra.munoz@ iztacala.unam.mx
} 
similares, así como de obtener otras medidas de validez y confiabilidad para tener la certeza de que efectivamente se está evaluando el constructo de interés.

Palabras Clave: aprendizaje autogestivo, validez, confiabilidad, educación en línea, universitarios.

\begin{abstract}
The objective of this research was to design and validate a scale of self-management learning in university students of an online system. For this purpose, we used an instrumental design divided into two phases; in the first phase 149 university students of the online modality were evaluated, through the strategy of modified natural semantic networks, to know the psychological meaning of the stimuli presented. The most recurrent defining words were: organization, self-taught, commitment, satisfaction, responsibility; which served as the basis for designing the proposal of an instrument aimed to evaluate self-managed learning on a Likert scale with five response options. In the second phase, 929 university students from an online system participated, to whom the proposal was applied and the psychometric properties of the scale were obtained. An exploratory factor analysis with varimax rotation was performed, which resulted in six factors (negative affect for online learning, aptitude for self-managed learning, responsibility in the learning process, actions to learn independently, technological resources for learning and personal resources for learning), which explain the $59.74 \%$ of variance, and a reliability of .846 . The results are discussed in terms of the importance of replicating this instrument to similar samples, as well as obtaining other measures of validity and reliability to be convinced that the construct of interest is actually being evaluated.
\end{abstract}

Keywords: self-management learning, validity, reliability, online education, university students.

\section{INTRODUCCIÓN}

El siglo XXI está enmarcado en cambios sustanciales a nivel global, en todos los aspectos en los que los individuos se desempeñan, habiendo hecho que estos cambien sus perfiles, en un mundo donde lo presencial va unido a lo virtual, de lo cual no está exenta la educación al haber cimentado la educación a distancia y con ello cambiar los roles de los estudiantes, quienes se han visto obligados a gestionar su autoaprendizaje, desde esta perspectiva (Guarneros-Reyes, Espinoza-Zepeda, Silva \& Sánchez-Sordo, 2016; Littlejohn, Hood, Milligan \& Mustain, 2016; Yang, 2016) refieren que el aprendizaje autogestivo es la capacidad que posee el estudiante por administrar su proceso de aprendizaje, donde no sólo implica el proceso de autonomía y autorregulación, sino que además monitorea sus objetivos académicos, sus estrategias cognitivas, motivacionales y de apoyo para construir su conocimiento.

Sobre estos procesos, se menciona que el aprendizaje autónomo es aquel en el que la persona define lo que hará en su proceso de aprendizaje, es decir dónde, qué, cuándo y cómo va a estudiar (Cárcel, 2016; Enríquez-Negrete, Arias-García, Sánchez-Medina \& Oseguera-Jiménez, 2018), por su parte el aprendizaje autorregulado, no sólo se caracteriza por la capacidad de la persona para aplicar sus estrategias de aprendizaje; sino en la valoración que hace sobre su propio proceso de aprendizaje (Broadbent \& Poon, 2015; Torrano, Fuentes \& Soria, 2017); 
Siendo necesario que los estudiantes desarrollen esta habilidad de autogestión de su propio aprendizaje Wong et al. (2019) hace hincapié como estrategia que los cursos en línea masivos y abiertos, mejor conocidos como MOOC (Massive Open Online Course, por sus siglas en inglés) permiten que el aprendizaje tenga lugar en cualquier momento y en cualquier lugar, sin la necesidad de contar con un tutor que esté monitoreando el aprendizaje de los alumnos, es este último quien tiene un papel central en su propio proceso que lo obliga a tomar decisiones relacionadas con sus propias actividades de aprendizaje para lograr el éxito académico; de tal forma que se espera que quienes se inscriban a este tipo de cursos respondan a lo que se solicita y no sólo esperar a que se les diga qué deben y cómo hacerlo, que es una de las características que suelen encontrarse en algunos estudiantes, (Lee, Choi \& Cho, 2019).

Investigaciones al respecto han descrito que si bien la presencia del tutor es necesaria para el éxito de la educación en línea (Song, Kim \& Park, 2019), cuando se prescinde de esta figura se encuentra resultados diversos, como Alqurashi, (2019) que encontró una característica esencial para que los estudiantes terminen estos cursos con éxito, lo cual tenía que ver con la satisfacción del estudiante hacia los cursos en línea, Watson, Yu \& Watson, (2018) sobre la actitud percibida de los estudiantes sobre el tema y de la autoeficacia del usuario para aprender en sistemas en línea, (Meng et al., 2018). Otras investigaciones en cambio se centran sobre el proceso de aprendizaje, ya sea autónomo (Pinto, Fernández-Pascual \& Marco, 2019; Yang, 2016) autorregulado (Kim, Yoon, Jo \& Branch, 2018; Maldonado-Mahauad, Pérez-Sanagustín, Kizilcec, Morales \& Munoz-Gama, 2018; Vrieling, Stijnen \& Bastiaens, 2018) o autogestivo (Beach, 2017; Ponce, 2016).

Si bien los procesos de autonomía y autorregulación han sido ampliamente estudiados, contar con un instrumento que evalúe el aprendizaje autogestivo es prácticamente bajo; en inglés existen diversas escalas al respecto que al evaluar el aprendizaje auto-dirigido en algunos de sus componentes se evalúa la autogestión (Behar-Horenstein, Beck \& Su 2018; Cadorin, Cheng \& Palese, 2016; Demircioglu et al., 2018; Kima \& Lee,
2018); y en español, existe una versión chilena que traduce un instrumento anglosajón (Cerda \& Saiz, 2015), de tal forma que de acuerdo con Reyes-Lagunes (1993) es necesario contar con instrumentos que sean culturalmente válidos, por lo que el objetivo del presente trabajo es diseñar y validar una escala de aprendizaje autogestivo en estudiantes universitarios de un sistema en línea.

\section{Aprendizaje autogestivo}

Los espacios virtuales para el aprendizaje son cada vez más amplios y diversas universidades los han implementado para garantizar una mayor cobertura; que por la ubicación geográfica pueden hacer difícil su acceso a la educación, (Barclay, Donalds \& Osei-Bryson, 2018; Janakiraman, Watson \& Watson, 2018); una de estas modalidades tiene que ver con la creación de cursos MOOC, estos cursos tienen como característica principal que los estudiantes son responsables de su propio proceso de aprendizaje en donde no cuentan con un tutor al frente que les indique lo que deben hacer, (Watson, et al., 2018).

Una de las críticas a este tipo de cursos tiene que ver con la discrepancia entre quienes se inscriben y quienes terminan, por lo que se vuelve un desafío para el estudiante que desea aprender en línea bajo esta modalidad (Cho, \& Yoo, 2017; Wong et al., 2019). Diversas investigaciones resaltan que los estudiantes requieren de un esfuerzo adicional para tener éxito (Alqurashi, 2019; Moon-Heum, Yanghee \& DongHo, 2017; Rhode, Richter \& Miller, 2017), sin embargo, independiente de ello la percepción que los estudiantes tienen sobre su aprendizaje es muy importante (Roulston, Pope, Paulus \& deMarrais, 2018), e incluso quienes carecen de autodisciplina, administración del tiempo y habilidades de organización pueden tener dificultades para participar en este tipo de cursos (Fung, Yuen \& Yuen, 2018). De tal forma que como mencionan Fındık-Coşkunçay Alkış \& Özkan-Yıldırım (2018) la gestión del aprendizaje ha adquirido un papel importante en la educación.

En el caso particular de la modalidad en línea sin presencia de un tutor, cobra aún mayor relevancia, debido a que no existe una figura que moni- 
toree el proceso de aprendizaje del estudiante, de tal forma que éste debe ser capaz de gestionarlo (Buhl \& Andreasen, 2018). Sobre este punto se ha encontrado que quienes tienen una buena capacidad para planificar, controlar y gestionar su proceso de aprendizaje, tenderán a aprender más rápido y enfrentar las dificultades que se encuentre en su camino, (Khiat, 2017; Kizilcec, Pérez-Sanagustín \& Maldonado, 2017). Yamagata-Lynch, Do, Skutnik, Thompson, Stephens \& Tays (2015) señalan que este tipo de aprendizaje enfatiza como los estudiantes pueden sentirse capacitados para asumir su propia responsabilidad para decidir qué y cómo aprender; además de las habilidades tecnológicas que cuenten para lograrlo, (Sumuer, 2018).

Por lo que se entiende, el aprendizaje autogestivo como la capacidad del estudiante para, gestionar la forma en que aprende, a través del uso de diversos recursos de aprendizaje y herramientas tecnológicas; las cuales son mediadas por la valoración positiva que hace sobre su desempeño y de la motivación para aprender; todas estas acciones coadyuvarán para que el estudiante cuente con un proceso de toma de decisiones que le permitan hacer frente a cualquier situación de aprendizaje, de tal forma que logre los objetivos académicos.

\section{Evaluación del aprendizaje autogestivo}

Para la evaluación del aprendizaje autogestivo se han diseńado diversos instrumentos para evaluar las características de los estudiantes en sistemas en línea, uno de ellos ha sido Zimmerman \& Kulikowich, (2016) al desarrollar una escala para evaluar la autoeficacia hacia el aprendizaje en línea, que resultó en tres factores, aprendizaje en el entorno en línea, tiempo-gestión y uso de la tecnología; si bien el instrumento obtuvo propiedades psicométricas adecuadas, la evaluación está centrada en cómo perciben los estudiantes lo que hacen.

El estudio realizado por $\mathrm{Na}$, Wang \& Arterberry, (2015) estuvo enfocado en el desarrollo y validación de un inventario de aprendizaje autodirigido en el que se obtuvieron ocho factores: necesidades de aprendizaje, uso de habilidades, desafíos permanentes, autoeficacia en el aprendizaje, habi- lidades de planificación, evaluación de habilidades, completar tareas y atribución interna, si bien obtuvieron propiedades psicométricas adecuadas, la muestra fueron mujeres escolarizadas que no pertenecían a un sistema en línea, por lo que esta experiencia o habilidades que se deben poseer son diferentes, tal y como lo señala Sumuer, (2018).

Esto mismo sucede con el instrumento desarrollado y validado por Kima \& Lee (2018) centrado en el aprendizaje autodirigido en estudiantes de primaria; utilizaron un análisis factorial exploratorio y confirmatorio, el alfa de Cronbach fue de .944 que si bien es alto, se corre el riesgo de que haya una sobrerrepresentación de ítems que es necesario hacer una nueva revisión, para tal vez omitir alguno de ellos (Nunnally, 1987); sin embargo se rescata la idea de que los reactivos se clasificaron en tres dominios; cognición (pensamiento cognitivo, metacognición y resolución de problemas), afectivo (motivación intrínseca, motivación orientada al futuro y autoeficacia), y comportamiento (búsqueda de ayuda, manejo físico - medio ambiente y gestión del tiempo).

En cuanto a la escala de aprendizaje autodirigida fue traducida del inglés al turco por Demircioglu et al., (2018) en la que se encontró buenas propiedades psicométricas, dicho instrumento es unidimensional y se compone de 10 reactivos; Chianchana (2016), por su parte validó la escala sobre las características del aprendizaje autodirigido, donde obtuvo ocho factores: apertura a oportunidades de aprendizaje, auto-concepción como un aprendiz efectivo, iniciativa e independencia en aprendizaje, aceptación informada de la responsabilidad del propio aprendizaje, amor por el aprendizaje, creatividad, una orientación positiva hacia el futuro y la capacidad de usar habilidades de estudio básicas y resolución de problemas, una de las dificultades de estas dos validaciones es que fueron hechas en personas que asisten a un sistema presencial y no en personas que estén en un sistema de aprendizaje en línea.

En población latina Cerda \& Saiz (2015) validaron la Escala de Aprendizaje Autodirigido en una muestra de estudiantes universitarios, encontrando propiedades psicométricas adecuadas y obteniendo tres factores (autocontrol, autogestión y 
deseos de aprender), la dificultad de retomar este instrumento primero es que es una versión traducida y segundo, que al igual que se ha señalado, la validación del instrumento se realizó con una muestra que estudia contextos presenciales.

Derivado del análisis presentado surgen las siguientes conclusiones que ponen en relieve la necesidad de construir un instrumento que permita evaluar las experiencias sobre el aprendizaje autogestivo:

1. Aprendizaje autodirigido no es sinónimo de aprendizaje autogestivo.

2. Los instrumentos sólo consideran algunos indicadores de la autogestión; por lo que hasta el momento no se cuenta con instrumentos que evalúen el aprendizaje autogestivo en su totalidad

3. Un gran número de instrumentos son validados en poblaciones diferentes a la latina, por lo que pudiera no existir correspondencia entre culturas.

4. El único instrumento validado en población latina es una traducción de un instrumento perteneciente a otro contexto sociocultural.

5. La mayoría de los instrumentos están desarrollados para evaluar estudiantes de sistemas presenciales, por lo que no se considera el medio tecnológico como elemento de la autogestión.

6. La validación de los instrumentos se ha realizado con muestras que estudian en sistemas presenciales, que, si bien comparten características con los estudiantes de ambientes virtuales, también difieren, por lo que estas características no se contemplan en las mediciones.

Con base en lo anterior se propone el diseño de un instrumento de medición utilizando la estrategia de Redes Semánticas Naturales Modificadas (RSNM) de Reyes-Lagunes, (1993) quien señala que dentro del proceso de construcción de instrumentos no basta con hacer una revisión de la literatura para el diseño de los reactivos, sino que además si se desea construir un instrumento culturalmente válido se debe preguntar a las personas sobre lo que piensan, hacen y sienten, de tal forma que se cuenten con indicadores más fieles al momento de plantear los reactivos.
Con base en la literatura se consideró el uso de cuatro estímulos (frases); con la finalidad de obtener indicadores del aprendizaje autogestivo de los propios estudiantes bajo esta modalidad, considerando tres dimensiones, cognitiva, emocional y conductual; que, de acuerdo con Martínez, Hernández \& Hernández, (2006) para tener una mejor validez de un instrumento se debe tener una representación de todas las dimensiones del constructo que se desea evaluar.

\section{Materiales Y Métodos}

Se recolectó la información en dos momentos debido a que la investigación se divide en dos fases, en un primer momento para la construcción del instrumento de medición y en un segundo momento para realizar la validación.

\section{Participantes}

Para la construcción del instrumento de medición la población estuvo conformada por estudiantes de la carrera de psicología de una universidad pública, pertenecientes a un sistema de educación en línea. Participaron 149 estudiantes a través de un muestreo intencionado (Kerlinger $\&$ Lee, 2002), se invitó a estudiantes pertenecientes al sector urbano de primero a noveno semestre a participar en la investigación. Como criterio de inclusión se consideró que estuvieran inscritos en la licenciatura y al menos estar cursando una materia al momento de responder el cuestionario, como criterio de exclusión, se descartaron a quienes no respondieron completo el cuestionario. El $18.79 \%$ fueron hombres y el $81.21 \%$ mujeres, todos ellos con una media de edad de 40.24 años $(\mathrm{SD}=11.98)$; $45 \%$ solteros, $30 \%$ casados $18 \%$ en una unión libre y el resto divorciados. El $87 \%$ de los participantes reporta trabajar en promedio 8 horas y dedicar 4.37 horas a sus actividades académicas. El $40 \%$ de la muestra está inscrito en los primeros cuatro semestres de la carrera.

En el caso de la validación del instrumento de medición la población estuvo conformada por 3644 estudiantes inscritos durante un semestre lectivo en la Licenciatura en Psicología en su modalidad a distancia, pertenecientes al sector urbano. Par- 
ticiparon 929 estudiantes $(25.49 \%$ del total de la población). Como criterio de inclusión debían estar inscritos en al menos una materia al momento de responder el cuestionario, como criterios de exclusión se descartó a quienes no respondieron completo el instrumento. $26 \%$ son hombres y $74 \%$ mujeres, con una edad promedio de 37.30 años $(\mathrm{SD}=10.62), 38 \%$ se encontraba soltero, $52 \%$ casado y el resto divorciado. El $74 \%$ de los participantes reporta trabajar en promedio 8.27 horas al día y dedicar 4.27 horas a sus actividades académicas. $64 \%$ de la muestra estaba inscrito en los primeros cuatro semestres de la carrera.

\section{Instrumento}

Escala de aprendizaje autogestivo en educación a distancia (EAA-ED). Consta de 43 afirmaciones que describen situaciones que todo estudiante puede experimentar al aprender en un sistema no presencial, con cinco opciones de respuesta (nunca, casi nunca, a veces, casi siempre, siempre) en formato tipo Likert. La indicación fue que debía marcar la opción que mejor describiera lo que hace durante su proceso. (Anexo 2).

\section{Tipo y diseño}

El tipo de estudio es instrumental debido a que está centrado en el análisis de las propiedades psicométricas de una escala de medición de acuerdo con Montero \& León (2007). El tipo de diseño de investigación es transversal (Kerlinger \& Lee, 2002), ya que únicamente se recolectan datos en un sólo momento.

\section{Procedimiento}

Fase 1. Construcción del instrumento de medición

Para la construcción del instrumento se solicitó apoyo a la Coordinación de Educación a Distancia para el envío de los mensajes a través de la plataforma de aprendizaje que utilizan los estudiantes para cursar la licenciatura en línea de Psicología. Se invitó a estudiantes de primero a noveno semestre a participar en la investigación a través de un mensaje personalizado.

En el cuerpo del mensaje se anexaba un link que los dirigía a un formulario que debían responder, el cual tardaron 20 minutos en contestarlo aproximadamente. Se utilizó la estrategia de Redes Semánticas Naturales Modificadas (RSNM) de Reyes-Lagunes (1993); esta autora propone que un instrumento de medida debe obtener una muestra representativa de conductas en las que se refleje las propiedades de lo que se desea medir, considerar las características culturalmente específicas de grupo a evaluar y que el significado tiene una función mediadora y tiene un papel importante en la medición; con base en estas ideas la estrategia de RSNM, se utiliza en el campo de la medición para: a) conocer el significado psicológico de uno o varios conceptos, y/o identificar aquellas conductas o indicadores que las personas consideran pertenecen al constructo de interés; de tal forma que no sólo basta con hacer una revisión teórica del constructo; sino que con esta estrategia se busca tener instrumentos más confiables, válidos y culturalmente relevantes. De acuerdo con la literatura revisada respecto al constructo de aprendizaje autogestivo, se eligieron cuatro estímulos (frases), los cuales se presentaron de manera aleatoria, solicitando se definiera con al menos cinco palabras que considerara relevantes y que después los numeraran en orden de importancia (Anexo 1). Los estímulos utilizados fueron: a) habilidades de un estudiante en línea, b) estrategias de estudio en educación a distancia, c) aprender en línea y d) experiencias de aprendizaje en línea.

Para el análisis de la información se utilizó una hoja de cálculo en el programa Excel (MS Office 2017); con cada una de las palabras definidas en cada uno de los estímulos, se realizó un análisis de frecuencias y una ponderación en términos de la importancia que ellos atribuían a cada palabra, con estos datos se obtuvo el peso semántico (PS) y la distancia semántica cuantitativa (DSC), esta información permitió obtener el significado que los estudiantes atribuyeron a cada estímulo en términos de la importancia que tiene para ellos.

Posterior a este análisis, como lo señala la RSNM se realizó una propuesta de reactivos que contemplan las palabras definidoras con mayor PS que 
los participantes mencionaron en función de los estímulos; para proceder a la segunda fase.

\section{Fase 2. Validación del instrumento de medición}

Previa autorización de la Coordinación de Educación a Distancia a toda la comunidad universitaria se le envió un mensaje a través de la plataforma de aprendizaje, que es el espacio donde los estudiantes realizan sus actividades académicas y formativas; se les invitó a responder una escala, a quienes desearon colaborar se les envío un formulario en línea con la escala. En promedio tardaron en responder 20 minutos.

Para realizar la validación, las respuestas del instrumento se vaciaron en el programa SPSS v24. Como primer paso se realizó un análisis del poder discriminativo de los reactivos a través de una prueba t de student para datos no relacionados, tomando como punto de referencia las puntuaciones obtenidas en los cuartiles Q1 y Q3; posteriormente se aplicó la prueba de esfericidad de Barlett y la medida de adecuación muestral KMO para identificar la conveniencia de realizar un análisis factorial, así como la pertinencia de la muestra utilizada para los análisis. Con base en estos resultados, se optó por realizar un análisis factorial exploratorio de componentes principales con rotación varimax, considerando cargas factoriales por encima de .4; por último, se obtuvo la consistencia interna de cada uno de los factores obtenidos, así como de la escala en general.

\section{Confidencialidad o Consentimiento informado}

En cada una de las fases se les invitó a los estudiantes a responder un cuestionario haciendo énfasis en el anonimato y confidencialidad de la información, así como en la participación voluntaria. En ambos formularios se explicitó a través de una pregunta en la que debían responder afirmativamente para acceder al cuestionario (Anexo 1 y 2).

\section{Resultados}

Los resultados se presentan en dos momentos debido a que la investigación se divide en dos fases, en un primer momento se describirán los aspectos recabados de la RSNM para la construcción del instrumento de medición y en un segundo momento los datos obtenidos de la validación y confiabilidad del instrumento.

Como producto de la fase de construcción del instrumento se presentan las palabras definidoras derivadas de la estrategia de RSNM para cada uno de los cuatro estímulos utilizados, reportando aquellos descriptores con mayor PS.

En la Figura 1, se observan las principales palabras descriptoras que los universitarios le atribuyen al estímulo "habilidades de un estudiante en línea”, entre más cercano al cero, significa que tiene mayor significado y conforme se va alejando lo va perdiendo. Los estudiantes perciben que dentro de éstas se engloban las actividades como: lecturas, resumen, mapas, subrayar; además de las acciones a ejecutar para lograrlo como organización, tiempo, planeación entre otras.

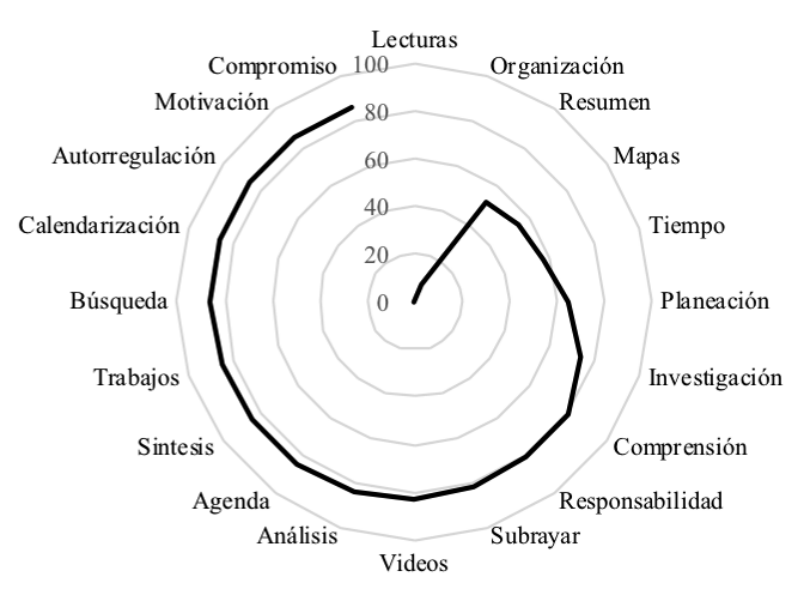

Figura 1

Descriptores del estímulo "Habilidades de un estudiante en línea”.

En cuanto al estímulo "estrategias de estudio en educación a distancia", los estudiantes reportan que éste se describe por palabras como: organización, responsabilidad, ser autodidactas, disciplinados, comprometidos (Figura 2). 


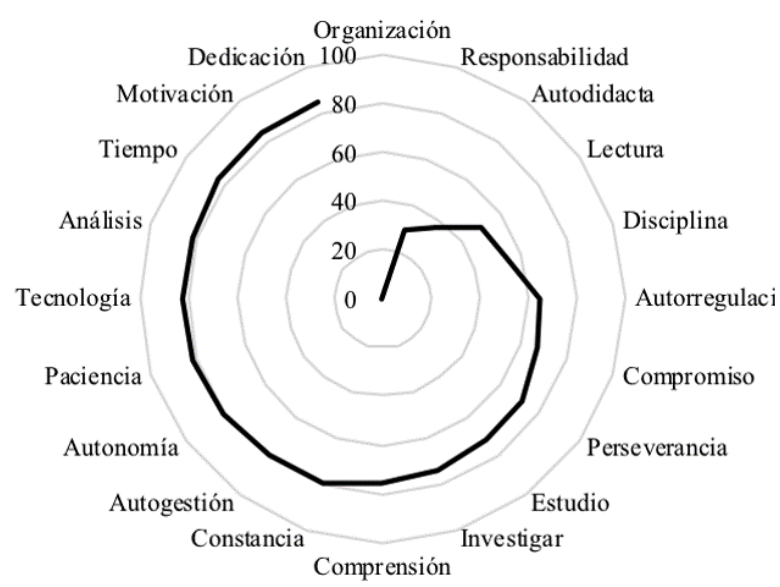

Figura 2

Descriptores del estímulo "Estrategias de estudio en educación a distancia”.

Sobre el estímulo "Aprender en Línea", la valoración de los estudiantes es en términos generales más positiva que negativa, como palabras descriptoras negativas mencionan complicado y difícil; en tanto que los descriptores positivos fueron: oportunidad, reto, satisfactorio, compromiso entre otras (ver Figura 3).

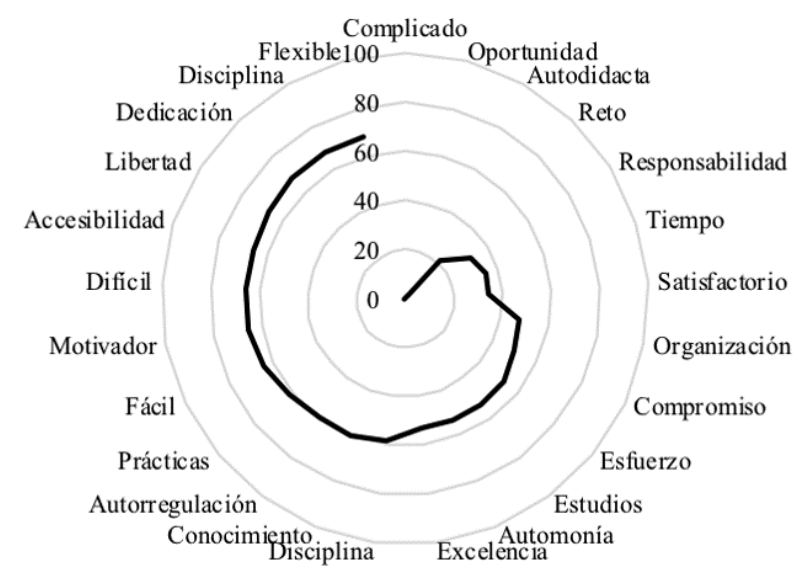

Figura 3

Descriptores del estímulo "Aprender en línea”.

Por último, en la Figura 4 se observan las palabras definidoras sobre el estímulo "experiencias de aprendizaje en línea”, los universitarios coinciden en palabras como: satisfacción, aprendizaje, retos, conocimientos, difícil, estresante y frustrante.

Considerando la estrategia de RSNM se retomaron las palabras definidoras mencionadas por los universitarios para cada uno de los estímulos presentados y se redactaron 43 reactivos que conformaron un instrumento inicial para evaluar el aprendizaje autogestivo.

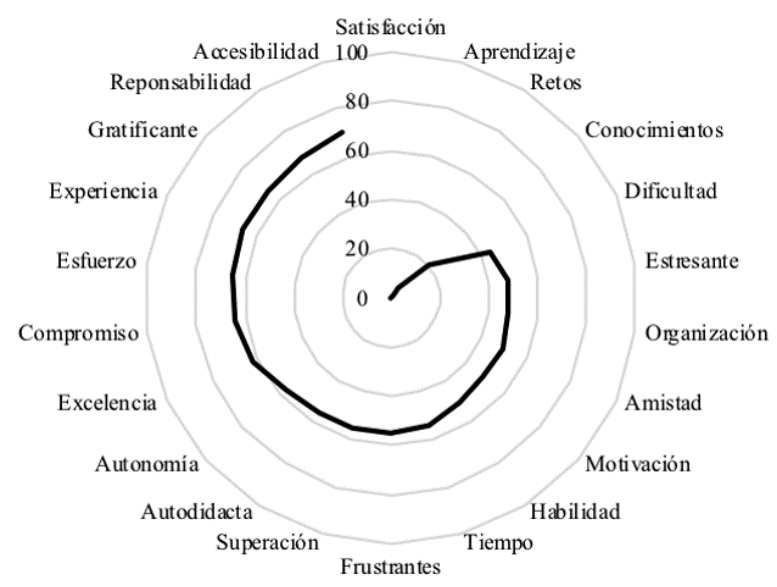

Figura 4

Descriptores del estímulo "Experiencias de aprendizaje en línea”.

Para la segunda fase, se aplicó la propuesta inicial del instrumento, a partir de los datos obtenidos se realizó la validación y confianza, que se describen a continuación.

Como resultado del proceso de poder discriminativo de los reactivos, en la Tabla 1, se observa que hubo diferencias significativas entre los grupos algo y bajo, respecto a las puntuaciones obtenidas en cada uno de los reactivos, por lo que los 43 reactivos fueron considerados en el análisis factorial.

\section{Tabla 1}

Poder discriminativo de cada uno de los reactivos de la EAA-ED

\begin{tabular}{|c|c|c|c|c|c|c|c|}
\hline $\begin{array}{l}\mathrm{Re}- \\
\text { activo }\end{array}$ & $\begin{array}{l}\text { Gpo. } \\
\text { Bajo } \\
n= \\
245\end{array}$ & DE & $\begin{array}{l}\text { Gpo. } \\
\text { Alto } \\
n= \\
248\end{array}$ & $\mathrm{DE}$ & $t$ & $\mathrm{gl}$ & $P$ \\
\hline 1 & 4.56 & .664 & 4.97 & .218 & 6.958 & 491 & .001 \\
\hline 2 & 4.07 & .813 & 4.86 & .467 & 10.005 & 491 & .001 \\
\hline 3 & 4.43 & .616 & 4.92 & .292 & 8.705 & 491 & .001 \\
\hline 4 & 3.94 & .832 & 4.83 & .392 & 11.574 & 491 & .001 \\
\hline 5 & 3.18 & 1.053 & 3.69 & .887 & 4.459 & 491 & .001 \\
\hline 6 & 4.25 & .933 & 4.76 & .491 & 5.734 & 491 & .001 \\
\hline 7 & 3.88 & .826 & 4.41 & .714 & 5.726 & 491 & .001 \\
\hline 8 & 3.90 & .806 & 4.50 & .637 & 6.879 & 491 & .001 \\
\hline 9 & 2.16 & .913 & 2.96 & 1.217 & 6.169 & 491 & .001 \\
\hline
\end{tabular}




\begin{tabular}{|c|c|c|c|c|c|c|c|}
\hline $\begin{array}{l}\mathrm{Re}- \\
\text { activo }\end{array}$ & $\begin{array}{l}\text { Gpo. } \\
\text { Bajo } \\
n= \\
245\end{array}$ & DE & $\begin{array}{l}\text { Gpo. } \\
\text { Alto } \\
n= \\
248\end{array}$ & DE & $t$ & $\mathrm{gl}$ & $P$ \\
\hline 10 & 2.15 & 1.072 & 2.95 & 1.155 & 6.032 & 491 & .001 \\
\hline 11 & 2.04 & .988 & 2.92 & 1.209 & 6.591 & 491 & .001 \\
\hline 12 & 3.78 & .867 & 4.77 & .512 & 11.733 & 491 & .001 \\
\hline 13 & 3.99 & .869 & 4.77 & .484 & 9.413 & 491 & .001 \\
\hline 14 & 3.94 & .718 & 4.85 & .373 & 13.460 & 491 & .001 \\
\hline 15 & 3.74 & .770 & 4.71 & .527 & 12.306 & 491 & .001 \\
\hline 16 & 4.25 & .653 & 4.93 & .255 & 11.611 & 491 & .001 \\
\hline 17 & 3.78 & .747 & 4.83 & .426 & 14.596 & 491 & .001 \\
\hline 18 & 4.02 & .821 & 4.92 & .267 & 12.503 & 491 & .001 \\
\hline 19 & 4.01 & .720 & 4.92 & .324 & 13.644 & 491 & .001 \\
\hline 20 & 3.82 & .854 & 4.67 & .603 & 9.670 & 491 & .001 \\
\hline 21 & 3.69 & .890 & 4.61 & .649 & 9.919 & 491 & .001 \\
\hline 22 & 4.16 & .752 & 4.87 & .340 & 10.216 & 491 & .001 \\
\hline 23 & 3.70 & .782 & 4.67 & .615 & 11.549 & 491 & .001 \\
\hline 24 & 3.69 & .839 & 4.78 & .508 & 13.192 & 491 & .001 \\
\hline 25 & 4.95 & .216 & 4.95 & .216 & 9.345 & 491 & .001 \\
\hline 26 & 3.52 & 1.040 & 4.51 & .869 & 8.618 & 491 & .001 \\
\hline 27 & 3.82 & .800 & 4.82 & .453 & 13.005 & 491 & .001 \\
\hline 28 & 4.16 & .722 & 4.92 & .292 & 11.689 & 491 & .001 \\
\hline 29 & 4.20 & .758 & 4.85 & .398 & 9.033 & 491 & .001 \\
\hline 30 & 3.48 & 1.142 & 4.31 & 1.033 & 6.367 & 491 & .001 \\
\hline 31 & 4.76 & .505 & 4.76 & .505 & 12.836 & 491 & .001 \\
\hline 32 & 2.11 & 1.016 & 3.08 & 1.271 & 7.051 & 491 & .001 \\
\hline 33 & 1.90 & .934 & 2.36 & 1.336 & 3.297 & 491 & .001 \\
\hline 34 & 3.90 & .837 & 4.85 & .398 & 12.239 & 491 & .001 \\
\hline 35 & 3.64 & .840 & 4.81 & .414 & 14.847 & 491 & .001 \\
\hline 36 & 3.85 & .830 & 4.85 & .373 & 13.134 & 491 & .001 \\
\hline 37 & 2.54 & 1.135 & 3.19 & 1.206 & 4.644 & 491 & .001 \\
\hline 38 & 3.65 & .812 & 4.72 & .599 & 12.572 & 491 & .001 \\
\hline 39 & 3.04 & 1.003 & 3.50 & 1.031 & 3.748 & 491 & .001 \\
\hline 40 & 2.43 & 1.009 & 3.04 & 1.194 & 4.589 & 491 & .001 \\
\hline 41 & 3.77 & .779 & 4.34 & .768 & 6.145 & 491 & .001 \\
\hline 42 & 3.64 & .814 & 4.71 & .613 & 12.457 & 491 & .001 \\
\hline 43 & 3.31 & .784 & 4.38 & .766 & 11.579 & 491 & .001 \\
\hline
\end{tabular}

Fuente: Elaboración propia (2019).

Con la finalidad de identificar la pertinencia del análisis factorial y del tamaño muestral, se realizó la prueba de esfericidad de Bartlett y KMO resultando ambas pruebas significativas (.919, $\mathrm{p}<.001)$; con estos datos se procedió a realizar un análisis factorial exploratorio. En la Tabla 2, se presentan los valores obtenidos de dicho análisis, de los 43 reactivos iniciales se eliminaron 9 que no se ajustaron a ningún factor, quedando 34 reactivos en el instrumento. Derivado del análisis se encontraron seis factores que explican el 59.74\% de la varianza, los cuales se nombraron de acuerdo a los aspectos que evaluaban de forma conjunta y retomando la revisión de la literatura respecto al aprendizaje autogestivo. Finalmente, de la prueba alfa de Cronbach para evaluar la consistencia interna se obtuvo un índice de .846 .

\section{Tabla 2}

Resultados del análisis factorial y consistencia interna de la EAA-ED

\begin{tabular}{lllllll}
\hline \multirow{2}{*}{$\begin{array}{l}\text { Número de reactivo y } \\
\text { factores }\end{array}$} & 1 & 2 & 3 & 4 & 5 & 6
\end{tabular}

Factor 1. Afecto negativo para el aprendizaje en línea

11. Cuando las instruc0.852 ciones de la actividad son confusas afecta mi estado de ánimo.

10. Cuando desconozco el $\quad 0.843$ tema de la actividad afecta mi estado de ánimo.

32. Cuando me siento 0.824 incomprendido por parte del tutor me estreso.

40. Experimento estrés $\quad 0.702$ durante mi aprendizaje.

37. Cuando me frustro se ve 0.682 afectado mi aprendizaje.

9. Me provoca ansiedad la $\quad 0.675$ falta de comunicación con el tutor.

33. Me genera frustración $\quad 0.674$ las actividades de aprendizaje.

5. Me estreso al tener cerca- 0.639 nas las fechas de entrega de las actividades.

39. Mi cansancio afecta $\quad 0.568$ el aprendizaje que debo adquirir.

Factor 2. Aptitud para el aprendizaje autogestivo

42. La disciplina forma parte 0.719 de mis habilidades.

34. Soy perseverante en mis $\quad 0.702$ actividades académicas.

27. Soy constante para concretar mi aprendizaje.

28. Me comprometo para lograr un buen aprendizaje. 


\begin{tabular}{lllllll}
\hline Número de reactivo y & \multicolumn{7}{c}{ Carga factorial } \\
\cline { 2 - 7 } factores & 1 & 2 & 3 & 4 & 5 & 6
\end{tabular}

16. Soy responsable en mis $\quad 0.596$

estudios.

36. Mi deseo de superación

0.442 me lleva a buscar la exce-

lencia académica.

\section{Factor 3. Responsabilidad en el proceso de aprendizaje}

19. El tiempo que dedico a

0.702

la realización de actividades me permite desarrollar habilidades.

18. El tiempo que dedico al estudio me lleva a adquirir habilidades.

22. Adquiero conocimientos cuando realizo investigación bibliográfica.

17. El tiempo que dedico como estudiante en línea me ayuda a desarrollar habilidades tecnológicas para realizar mis actividades.

20. Mis habilidades de comunicación influyen en mi aprendizaje.

21. Mi creatividad es imprescindible en la realización de las actividades académicas.

\section{Factor 4. Estrategias para aprender de manera independiente}

23. Soy autodidacta.

31. Soy autodidacta para

lograr aprendizajes.

15. Soy autodidacta para alcanzar los aprendizajes.

35. La autonomía es una de mis estrategias de aprendizaje.

\section{Factor 5. Recursos tecnológicos para el aprendizaje}

13. Cuento con habilidades tecnológicas para estudiar en línea.

8. Tengo un manejo adecuado de la tecnología que simplifica el desarrollo de las actividades académicas.

14. La innovación tecnológica hace de mi aprendizaje una meta interesante.

\begin{tabular}{lllllll}
\hline \multirow{2}{*}{$\begin{array}{l}\text { Número de reactivo y } \\
\text { factores }\end{array}$} & 1 & 2 & 3 & 4 & 5 & 6 \\
\cline { 2 - 7 } & & & & & 0.628 \\
\hline $\begin{array}{l}\text { 6. Cuento con un equipo } \\
\text { de cómputo adecuado para } \\
\text { garantizar la realización de } \\
\text { mis actividades. }\end{array}$ & & & & & \\
\end{tabular}

\section{Factor 6. Recursos personales para el aprendizaje}

3. Analizar la información me facilita el aprendizaje.

12. Mi nivel de autonomía fortalece mi proceso de aprendizaje.

1. Leer el material del curso forma parte de mi aprendizaje.

4. Mi autorregulación me permite mantener el objetivo académico.

2. Ser organizado/a es clave en mi éxito como estudiante.

\begin{tabular}{rlllllll}
\hline Varianza explicada & 25.65 & 11.96 & 7.21 & 5.74 & 4.89 & 4.28 \\
Alfa de Cronbach & .894 & .846 & .805 & .872 & .775 & .720 \\
\hline \multicolumn{6}{c}{ Varianza explicada total: 59.74} \\
\multicolumn{6}{c}{ Alfa de Cronbach total: .846 } \\
\hline
\end{tabular}

Fuente: Elaboración propia (2019).

\section{Discusión y Conclusiones}

El objetivo de la presente investigación fue diseñar y validar una escala de aprendizaje autogestivo en estudiantes universitarios de un sistema en línea. Como parte de la revisión de la literatura se encontró que son pocos los instrumentos que evalúan el aprendizaje autogestivo en sistemas en línea, y quienes lo hacen, evalúan el aprendizaje autodirigido, en la que consideran solo algunas de sus dimensiones, (Behar-Horenstein, et al., 2018; Cadorin, et al., 2016; Demircioglu et al., 2018; Kima \& Lee, 2018).

El aprendizaje autogestivo, por lo tanto, es una característica esencial que debe poseer todo estudiante, sobre todo aquellos que están en un sistema en línea (Guarneros-Reyes et al., 2016) y más aún si dentro de estas modalidades se tienen cursos en los que la presencia del tutor no está 
disponible (Castaño, Maiz \& Garay, 2015), de tal forma que estas nuevas formas de aprender requieren que sea el alumno quien sea el principal actor en su proceso de aprendizaje, (Yang, 2016).

En estas modalidades existe una controversia respecto a su aplicabilidad y efectividad en el proceso de aprendizaje (Alqurashi, 2019; Watson et al., 2018), motivo por el cual es necesario poner atención en la forma en la que los estudiantes están aprendiendo para poder tener indicadores de qué lleva a un estudiante a culminar su formación en un sistema en línea.

Una de las características que se considera en este proceso tiene que ver con el aprendizaje autogestivo, de acuerdo con Littlejohn et al., (2016) este proceso implica que el estudiante haga uso de todos sus recursos para regular, monitorear y modificar su proceso de aprendizaje, de tal forma que le permita lograr sus objetivos académicos; sin embargo, una de las dificultades encontradas en la revisión de la literatura, es que si bien se habla de las características que debe tener para aprender de manera autogestiva, no existe suficiente evidencia empírica que contribuya con estas afirmaciones; de tal forma que es necesario contar con ello para poder contribuir con estos indicadores.

Si bien no se encontró en la revisión de la literatura un instrumento como tal que evaluará aprendizaje autogestivo, se optó por el diseño y construcción de uno; para ello se utilizó en un primer momento la estrategia de RSNM (Reyes-Lagunes, 1993), que tiene por objetivo conocer el significados psicológico del constructo al preguntar a las personas que piensan, sienten y hacen en relación a una serie de estímulos que se les presentan; de tal forma que se puede hacer un instrumento de medición que sea culturalmente válido y que contribuya a un mejor acercamiento al significado del constructo en un grupo específico de personas.

De acuerdo con Hinojosa (2008) el tamaño de la muestra para las RSNM debe ser determinado de igual forma que en cualquier investigación, este autor señala que se debe seguir respetando la propuesta original de tener al menos 50 personas para ello, cumpliendo con el criterio de saturación teórica; cabe señalar que diversas investigaciones que la usan obtienen buenos resultados en los instrumentos que se validan (Fonseca, Cruz \& Chacón, 2019; García-Torres, García-Méndez \& Rivera-Aragón, 2017; Granados, 2019; Santisteban-Negroe \& Reyes-Lagunes, 2018).

Con base en lo anterior, la aplicación de la estrategia de RSNM se realizó aproximadamente con 150 estudiantes universitarios, de tal forma que se puede tener certeza de que se tiene una representación del significado de lo que los estudiantes piensan, hacen y sienten respecto del aprendizaje autogestivo.

Sobre el significado del estímulo "habilidades de un estudiante en línea”, se encontró que los universitarios reportaron, lecturas, organización, tiempo, planeación, investigación, responsabilidad; que si bien pueden ser aspectos abstractos, también mencionaron actividades concretas como ver videos, agendar, y calendarizar, estos resultados sugieren que la parte de la planeación y organización son las características principales que deben poseer como estudiantes en línea, además que las lecturas fueron la principal palabra definidora, lo cual tiene relación con no tener a un profesor o tutor al frente, de tal forma que el medio con el que cuentan para aprender principalmente son las lecturas, (Flores, 2016).

Respecto al estímulo "estrategias de estudio", los estudiantes reportaron organización, responsabilidad, ser autodidactas, ser disciplinados, incorporan aspectos relacionados con la tecnología que difícilmente se obtendría si se consideraran estudiantes de sistemas presenciales (Cerda \& Saiz; 2015; Chianchana, 2016). Sobre el estímulo "aprender en línea", los participantes reportaron palabras como: complicado, difícil, oportunidad, reto, responsabilidad, tiempo, organización y compromiso; se observan tanto características positivas, como negativas que tiene que ver con los escenarios a los que están expuestos; y como se ha mencionado, si bien los sistemas en línea están diseñados para personas que por diversas razones no pueden acudir a un sistema presencial (Bartolomé $\&$ Steffens, 2015), las situaciones que van experimentando en estos sistemas se perciben como difíciles si los estudiantes no tienen las habilidades necesarias para regular su proceso de aprendizaje, (Broadbent \& Poon, 2015). 
En este mismo sentido, las palabras que definen el estímulo "aprendizaje en línea" estuvieron tanto en términos positivos, como negativos, ellos describen a través de: satisfacción, aprendizaje, retos; así como difícil, estresante y frustrante; esto es relevante, debido a que en muchas ocasiones el éxito o fracaso de estos sistemas tiene que ver con la falta de claridad de qué es lo que deben de hacer y el tiempo que dedican a ello, (Raposo-Rivas, Sarmiento \& Martínez-Figueira, 2017). Otros descriptores mencionados son: superación, autonomía y autodidacta, los cuales deben tomarse en cuenta para futuras investigaciones, ya que es lo que caracteriza a los estudiantes de la modalidad en línea, fomentando estos aspectos en los estudiantes desde las primeras aproximaciones a los sistemas en línea se pueden evitar desenlaces negativos como la frustración, el estrés y el abandono.

Con base en los resultados de RSNM se diseñó un instrumento para evaluar el aprendizaje autogestivo, a grosso modo se observa que se obtuvieron propiedades psicométricas adecuadas, aportando, por un lado, evidencia empírica del uso de la estrategia de RSNM propuesta por Reyes-Lagunes (1993) en el proceso de construcción de instrumentos en psicología y, por otro lado, contar con un instrumento válido y confiable para evaluar el aprendizaje autogestivo en universitarios de educación a distancia.

Derivado del análisis factorial el primer factor, está relacionado con aspectos negativos para aprender en línea, como no tener claridad de las instrucciones, no saber del tema, sentirse estresado, frustrado y cansado; si bien, no son características propias de un aprendizaje autogestivo, si se menciona que para que este proceso se dé, es necesario que el estudiante esté motivado y tenga una experiencia favorable (Broadbent $\& \mathrm{Fu}-$ ller-Tyszkiewicz, 2018), en el presente estudio los participantes mencionan que estos aspectos pueden afectar su aprendizaje en línea. Motivo por el cual se esperaría que los estudiantes en sistemas en línea disminuyan este afecto negativo para que logren concluir con éxito los cursos que tomen bajo esta modalidad.

El segundo factor que surgió del análisis estuvo centrado en la aptitud para aprender de mane- ra autogestiva, que se refiere a esa capacidad del estudiante para ser disciplinado, perseverante, constante, responsable y comprometido con lo que está aprendiendo, estas características definen a un estudiante que gestiona su propio aprendizaje, (Broadbent \& Poon, 2015; Xie, Hensley, Law $\&$ Sun, 2019).

El tercer factor, se relacionó con la responsabilidad del propio estudiante para aprender, donde se resaltaron características centradas en ser creativos, en el tiempo que dedican a sus actividades, como hacer investigación bibliográfica, que en su conjunto les permite mejorar su comunicación y manejo de la tecnología, todas estas características hacen referencia a la percepción que tiene el estudiante de lo que hace y cómo dedicarse al trabajo académico, tal y como menciona Torrano et al. (2017), es importante la valoración positiva que hace el estudiante sobre su propio proceso de aprendizaje.

El cuarto factor, estuvo centrado en las capacidades que poseen para aprender de manera independiente, siendo autodidactas para lograr y alcanzar su aprendizaje, esto es relevante, si se define como aquella acción de aprender por sí mismo, utilizando sus propios medios y sin ayuda de un profesor o tutor, y que es una parte esencial de alguien que aprende de manera autogestiva, (Behar-Horenstein et al., 2018; Cerda \& Saiz, 2015).

Los últimos dos factores, estuvieron centrados en los recursos tecnológicos y personales que poseen para el aprendizaje, respecto al primero, es relevante considerar que estos recursos tecnológicos son características que privilegian los estudiantes en sistemas en línea, ya que, si no poseen habilidades para desenvolverse en este medio, difícilmente lograrán el éxito académico, (Zimmerman \& Kulikowich; 2016). Los recursos personales están centrados en su capacidad de análisis, de ser autónomos, de leer y comprender el material de lectura, de autorregularse y ser organizados para el logro de sus objetivos, de tal forma que, como ya se mencionó el aprender de manera autogestiva, lleva consigo mismo los procesos de autorregulación y autonomía.

Con base en lo anterior, el instrumento puede ayudar a identificar las características autogestivas 
de los estudiantes en sistemas en línea, por lo que el siguiente paso es, por un lado, continuar con el proceso de validación y confiabilidad de este instrumento a través de otros métodos, por ejemplo, mediante la validez convergente o divergente que de sustento de que efectivamente se está evaluando lo que se desea (Post, 2016), a través del juicio de expertos que retroalimenten los reactivos y si los factores corresponden a lo que teóricamente se espera (Galicia, Balderrama \& Edel, 2017); realizar un análisis factorial confirmatorio que replique la estructura factorial y garantizar que estos factores están relacionados entre sí (Flora \& Flake, 2017), además de analizar la consistencia del instrumento en diferentes poblaciones (Heale \& Twycross, 2015).

En la primera fase por cada hombre que participó participaron cuatro mujeres, mientras que para la segunda fase la proporción fue de un hombre por cada tres mujeres. Debido a la naturaleza de la carrera es frecuente encontrar esta característica (ANUIES, 2018); lo cual invita a realizar un análisis respecto al perfil sociodemográfico de los participantes que contemple: sexo, contexto en el que se desarrollan, actividades a las que se dedican, tiempo para el estudio, situación familiar y de pareja, entre otros factores que permitan identificar si las características del aprendizaje autogestivo son diferentes en función de estas características.

Además, es necesario aplicar el instrumento a estudiantes universitarios de diferentes carreras que se ofertan en línea, para analizar sus similitudes y diferencias; tal y como lo reporta Freiberg, Ledesma \& Fernández (2017) quienes encontraron que la manera en la que aprenden los estudiantes varía en función de la carrera que están cursando.

En el caso particular de los estudiantes de psicología, también se ha hecho investigación para analizar sus estilos de aprendizaje (Bobadilla, Cardoso, Carreño \& Márquez, 2017), de tal forma que los resultados presentados hasta el momento corresponden a un tipo de estudiante universitario, por lo que es necesario, comparar la autogestión en función de la carrera, que permita contar con indicadores que nos permitan evaluar por que los estudiantes en un sistema en línea se rezaguen o deserten de sus estudios.
Por otro lado, podría aplicarse en cursos en línea con la presencia de tutor o no, con diferentes niveles de interacción entre compañeros, entre aquellos que terminan o no un curso bajo esta modalidad, y con base en ello, analizar las características autogestivas que poseen los estudiantes en línea, que permita generar estrategias para incrementar la eficiencia terminal y reducir el índice de reprobación de los estudiantes.

Se observan entonces, diversas líneas de investigación en las que se espera, por un lado, seguir contribuyendo con la confiabilidad de este instrumento, así como en la obtención de indicadores, que permitan explicar el aprendizaje autogestivo.

\section{Agradecimiento}

Trabajo realizado con el apoyo del Programa UNAM-DGAPA-PAPIME PE302819

\section{REFERENCIAS BIBLIOGRÁFICAS}

Alqurashi, E. (2019). Predicting student satisfaction and perceived learning within online learning environments. Distance Education, 40(1), 133-148. https://doi.org/10.1080/01587919.2018.1553562

ANUIES (2018). Anuario Estadístico de Educación Superior. Ciclo Escolar 2017-2018. Recuperado de http://www. anuies.mx/informacion-y-servicios/informacion-estadistica-de-educacion-superior/anuario-estadistico-de-educacion-superior

Barclay, C., Donalds, C., \& Osei-Bryson, K.-M. (2018). Investigating critical success factors in online learning environments in higher education systems in the Caribbean*. Information Technology for Development, 24(3), 582-611. https://doi.org/10.1080/02681102.2018.1476831

Bartolomé, A., \& Steffens, K. (2015). ¿Son los MOOC una alternativa de aprendizaje? Comunicar, 22(44), 91-99.

https://doi.org/10.3916/C44-2015-10

Beach, P. (2017). Self-directed online learning: A theoretical model for understanding elementary teachers' online learning experiences. Teaching \& Teacher Education, 61, 6072. https://doi.org/10.1016/j.tate.2016.10.007

Behar-Horenstein, L. S., Beck, D. E., \& Su, Y. (2018). An Initial Validation Study of the Self-Rating Scale of Self-Directed Learning for Pharmacy Education. American Journal of Pharmaceutical Education, 82(3), 280-286.

https://doi.org/10.5688/ajpe6251

Bobadilla, S, Cardoso, D., Carreño, L. \& Márquez, J. 
(2017). Estilos de aprendizaje en estudiantes de la licenciatura en psicología del centro universitario UAEM Temascaltepec, 2016. RIDE. Revista Iberoamericana para la Investigación y el Desarrollo Educativo, 7(14), 34-50.

https://doi.org/10.23913/ride.v7i14.271

Broadbent, J., \& Fuller-Tyszkiewicz, M. (2018). Profiles in self-regulated learning and their correlates for online and blended learning students. Educational Technology Research \& Development, 66(6), 1435-1455.

https://doi.org/10.1007/s11423-018-9595-9

Broadbent, J., \& Poon, W. L. (2015). Self-regulated learning strategies \& academic achievement in online higher education learning environments: A systematic review. Internet \& Higher Education, 27, 1-13.

https://doi.org/10.1016/j.iheduc.2015.04.007

Buhl, M., \& Andreasen, L. B. (2018). Upscaling the number of learners, fragmenting the role of teachers: How do massive open online courses (MOOCs) form new conditions for learning design? International Review of Education, 64(2), 179-195. https://doi.org/10.1007/s11159-018-9714-1

Cadorin, L., Cheng, S. F., \& Palese, A. (2016). Concurrent validity of self-rating scale of self-directed learning and self-directed learning instrument among Italian nursing students. Bmc Nursing, 15.

https://doi.org/10.1186/s12912-016-0142-x

Cárcel, F. (2016). Desarrollo de habilidades mediante el aprendizaje autónomo. 3C Empresa, 5(3). 52-60.

https://doi.org/10.17993/3cemp.2016.050327.52-60

Castaño, C., Maiz, I., \& Garay, U. (2015). Percepción de los participantes sobre el aprendizaje en un MOOC RIED. Revista Iberoamericana de Educación a Distancia, 18(2), 197-221. https://doi.org/10.5944/ried.18.2.13444

Cerda, C., \& Saiz, J. L. (2015). Aprendizaje autodirigido en estudiantes de pedagogía chilenos: un análisis psicométrico. Self-directed learning in Chilean student teachers: A psychometric analysis, 22(2), 129-136.

https://doi.org/10.1016/j.sumpsi.2015.08.004

Chianchana, C. (2016). Developing of the measurement Model of self-directed learning characteristics. In Z. Bekirogullari, M. Y. Minas, \& R. X. Thambusamy (Eds.), Iccsbs 2016 - the Annual International Conference on Cognitive - Social, and Behavioural Sciences (pp. 7-17). United Kingdom: Future Academy.

Cho, M.-H., \& Yoo, J. S. (2017). Exploring online students' self-regulated learning with self-reported surveys and log files: a data mining approach. Interactive Learning Environments, 25(8), 970-982.

https://doi.org/10.1080/10494820.2016.1232278

Demircioglu, Z. I., Oge, B., Fucular, E. E., Cevik, T., Nazligul, M. D., \& Ozcelik, E. (2018). Reliability, Validity and Turkish Adaptation of Self-Directed Learning Scale (SDLS). International Journal of Assessment Tools in Education, 5(2), 235-247. https://doi.org/10.21449/ijate.401069

Enríquez-Negrete, D., Arias-García, B., Sánchez-Medina,
R., \& Oseguera-Jiménez, O. (2018). Análisis longitudinal del desempeño académico de estudiantes de educación superior en un curso autónomo en línea. Revista Latinoamericana de Tecnología Educativa, 17(2), 101-115.

https://doi:10.17398/1695-288X.17.2.101

Fındık-Coşkunçay, D., Alkış, N., \& Özkan-Yıldırım, S. (2018). A Structural Model for Students' Adoption of Learning Management Systems: An Empirical Investigation in the Higher Education Context. Journal of Educational Technology \& Society, 21(2), 13-27. https://doi.org/10.1037/t70573-000

Flora, D. B., \& Flake, J. K. (2017). The purpose and practice of exploratory and confirmatory factor analysis in psychological research: Decisions for scale development and validation. Canadian Journal of Behavioural Science, 49(2), 78-88. https://doi.org/10.1037/cbs0000069

Flores, D. (2016). La importancia e impacto de la lectura, redacción y pensamiento crítico en la educación superior. Zona Próxima, (24), 128-135.

https://doi.org/10.14482/zp.22.5832

Fonseca, J., Cruz, C. \& Chacón, L. (2019). Validación del instrumento de compromiso organizacional en México: evidencias de validez de constructo, criterio y confiabilidad. Revista de Psicología, 37(1), 7-29.

https://doi.org/10.18800/psico.201901.001

Freiberg, A., Ledesma, R. \& Fernández, M. (2017). Estilos y estrategias de aprendizaje en estudiantes universitarios de Buenos Aires. Revista de Psicología (PUCP), 35(2), 535573. https://doi.org/10.18800/psico.201702.006

Fung, J. J. Y., Yuen, M., \& Yuen, A. H. K. (2018). Validity Evidence for a Chinese Version of the Online Self-Regulated Learning Questionnaire with Average Students and Mathematically Talented Students. Measurement and Evaluation in Counseling and Development, 51(2), 111-124.

https://doi.org/10.1080/07481756.2017.1358056

Galicia, L., Balderrama, J., \& Edel, R. (2017). Validez de contenido por juicio de expertos: propuesta de una herramienta virtual. Apertura, 9(2), 42-53. https://doi. org/10.32870/Ap.v9n2.993

García-Torres, M., García-Méndez, M., \& Rivera-Aragón, S. (2017). Apoyo social en adultos mexicanos: validación de una escala. Acta de investigación psicológica, 7(1), 25612567. https://doi.org/10.1016/j.aipprr.2017.02.004

Granados, R. (2019). Validación psicométrica de la Escala de Valoración de Riesgo de Violencia en Adolescentes. Archivos de Criminología, Seguridad Privada y Criminalista, 22, 107-121.

Guarneros-Reyes, E., Espinoza-Zepeda, A., Silva Rodríguez, A., \& Sánchez-Sordo, J. M. (2016). Diseño de un curso autogestivo modular en línea de metodología de la investigación para universitarios. Hamut'ay, 3(2), 7-24.

https://doi.org/10.21503/hamu.v3i2.1305

Heale, R., \& Twycross, A. (2015). Validity and reliability in quantitative studies. Evidence-Based Nursing, 18, 66-67. https://doi.org/10.1136/eb-2015-102129 
Hinojosa, G. (2008). El tratamiento estadístico de las redes semánticas naturales. Revista Internacional de Ciencias Sociales y Humanidades, 28 (1), 133-154.

Janakiraman, S., Watson, S. L., \& Watson, W. R. (2018). Adult learners use of self-directed learning strategies in a massive open online course. Journal of Ethnographic \& Qualitative Research, 13(2), 122-133.

Kerlinger, F., \& Lee, H. (2002). Investigación del comportamiento. Métodos de investigación en ciencias sociales. México: McGraw Hill.

Khiat, H. (2017). Academic performance and the practice of self-directed learning: The adult student perspective. Journal of Further \& Higher Education, 41(1), 44-59. https://doi.org/10.1080/0309877X.2015.1062849

Kim, D., Yoon, M., Jo, I.-H., \& Branch, R. M. (2018). Learning analytics to support self-regulated learning in asynchronous online courses: A case study at a women's university in South Korea. Computers \& Education, 127, 233-251. https://doi.org/10.1016/j.compedu.2018.08.023

Kima, S., \& Lee, K. (2018). Development and Validation of Self-directed Learning Ability Test (SDLAT) for Elementary School Students. International Electronic Journal of Elementary Education, 10(5), 551-557.

https://doi.org/10.26822/iejee.2018541304

Kizilcec, R. F., Pérez-Sanagustín, M., \& Maldonado, J. J. (2017). Self-regulated learning strategies predict learner behavior and goal attainment in Massive Open Online Courses. Computers \& Education, 104, 18-33.

https://doi.org/10.1016/j.compedu.2016.10.001

Lee, K., Choi, H., \& Cho, Y. H. (2019). Becoming a competent self: A developmental process of adult distance learning. Internet \& Higher Education, 41, 25-33.

https://doi.org/10.1016/j.iheduc.2018.12.001

Littlejohn, A., Hood, N., Milligan, C., \& Mustain, P. (2016). Learning in MOOCs: Motivations and self-regulated learning in MOOCs. Internet \& Higher Education, 29, 40-48. https://doi.org/10.1016/j.iheduc.2015.12.003

Maldonado-Mahauad, J., Pérez-Sanagustín, M., Kizilcec, R. F., Morales, N., \& Munoz-Gama, J. (2018). Mining theory-based patterns from Big data: Identifying self-regulated learning strategies in Massive Open Online Courses. Computers in Human Behavior, 80, 179-196.

https://doi.org/10.1016/j.chb.2017.11.011

Martínez, M., Hernández, M. \& Hernández, M. (2006). Psicometría. Madrid. Alianza Editorial.

Meng, M., Hey, T., Peter, D., Mattner, F., Igel, C., \& Kugler, C. (2018). From subjective self-assessment to objective behavior -- use and acceptance of a web 2.0 based e-learning structure in infection control. Von der subjektiven Einschätzung zum objektiven Verhalten -- Nutzung und Akzeptanz einer Web 2.0-basierten E-Learning-Struktur in Hygieneweiterbildungen, 14(3), 1-8.

https://doi:10.3205/mibe000193

Montero, I. \& León, O. (2007). A guide for naming research studies in Psychology. International Journal of Clini- cal and Health Psychology, 7(3), 847-862.

Moon-Heum, C., Yanghee, K., \& DongHo, C. (2017). The effect of self-regulated learning on college students' perceptions of community of inquiry and affective outcomes in online learning. The Internet and Higher Education, 34, 10-17. https://doi.org/10.1016/j.iheduc.2017.04.001

Na, H., Wang, K., \& Arterberry, B. (2015). Development and Initial Validation of the Self-Directed Learning Inventory with Korean College Students. Journal of Psychoeducational Assessment, 33(7), 687-697.

https://doi.org/10.1177/0734282914557728

Nunnally, J. (1987). Teoría psicométrica. México: Trillas

Pinto, M., Fernández-Pascual, R., \& Marco, F. J. G. (2019).

Self-learning of Information Literacy Competencies in Higher Education: The Perspective of Social Sciences Students. College \& Research Libraries, 80(2), 215-237. https://doi.org/10.5860/crl.80.2.215

Ponce, M. E. P. (2016). La autogestión para el aprendizaje en estudiantes de ambientes mediados por tecnología. Diálogos sobre educación, 7(12), 1-23.

Post, M. (2016). What to Do With "Moderate" Reliability and Validity Coefficients? Archives of Physical Medicine and Rehabilitation. 97(8). 1051-1052.

https://doi.org/10.1016/j.apmr.2016.04.001

Raposo-Rivas, M., Sarmiento, J., \& Martínez-Figueira, M. (2017). The pedagogic profile of the MOOC from an exploratory study. Estudios pedagógicos. 43(2), 277-292.

https://doi.org/10.4067/S0718-07052017000200015

Ravelo C, E. L. (2013). Descripción de factores sociodemográficos y socio afectivos y su relación con el desempeño académico de los estudiantes de cuarto semestre de psicología de una institución de educación superior. Psicogente, 16(29). Recuperado de http://revistas.unisimon.edu.co/index.php/psicogente/article/view/1938

Reyes-Lagunes, I. (1993). Redes semánticas para la construcción de instrumentos. Revista de Psicología Social y Personalidad. IX(1), 83-99.

Rhode, J., Richter, S., \& Miller, T. (2017). Designing Personalized Online Teaching Professional Development through Self-Assessment. TechTrends: Linking Research \& Practice to Improve Learning, 61(5), 444-451.

https://doi.org/10.1007/s11528-017-0211-3

Roulston, K., Pope, E., Paulus, T., \& deMarrais, K. (2018). Students' perceptions of learning about qualitative inquiry in online contexts. American Journal of Distance Education, 32(3), 190-201.

https://doi.org/10.1080/08923647.2018.1475921

Santisteban-Negroe, J., \& Reyes-Lagunes, L. (2018). La calidad de vida en adultos jóvenes mediante Redes Semánticas Naturales Modificadas. Informes Psicológicos, 18(2), 31 44. https://doi.org/10.18566/infpsic.v18n2a02

Song, H., Kim, J., \& Park, N. (2019). I Know My Professor: Teacher Self-Disclosure in Online Education and a Mediating Role of Social Presence. International Journal of 
Human-Computer Interaction, 35(6), 448-455.

https://doi.org/10.1080/10447318.2018.1455126

Sumuer, E. (2018). Factors related to college students' self-directed learning with technology. Australasian Journal of Educational Technology, 34(4), 29-43.

https://doi.org/10.14742/ajet.3142

Torrano, F., Fuentes, J. \& Soria, M. (2017). Aprendizaje autorregulado: estado de la cuestión y retos psicopedagógicos. Perfiles educativos, 39(156), 160-173.

https://doi.org/10.22201/iisue.24486167e.2017.156.58290

Vrieling, E., Stijnen, S., \& Bastiaens, T. (2018). Successful learning: balancing self-regulation with instructional planning. Teaching in Higher Education, 23(6), 685-700.

https://doi.org/10.1080/13562517.2017.1414784

Watson, W. R., Yu, J. H., \& Watson, S. L. (2018). Perceived attitudinal learning in a self-paced versus fixed-schedule MOOC. Educational Media International, 55(2), 170-181. https://doi.org/10.1080/09523987.2018.1484044

Wong, J., Baars, M., Davis, D., Van Der Zee, T., Houben, G.-J., \& Paas, F. (2019). Supporting Self-Regulated Learning in Online Learning Environments and MOOCs: A Systematic Review. International Journal of Human-Computer Interaction, 35(4/5), 356-373.

https://doi.org/10.1080/10447318.2018.1543084

Xie, K., Hensley, L. C., Law, V., \& Sun, Z. (2019). Self-regulation as a function of perceived leadership and cohesion in small group online collaborative learning. British Journal of Educational Technology, 50(1), 456-468.

https://doi.org/10.1111/bjet.12594

Yamagata-Lynch, L. C., Do, J., Skutnik, A. L., Thompson, D. J., Stephens, A. F., \& Tays, C. A. (2015). Design lessons about participatory self-directed online learning in a graduate-level instructional technology course. Open Learning, 30(2), 178-189.

https://doi.org/10.1080/02680513.2015.1071244

Yang, Y.-F. (2016). Self-directed learning to develop autonomy in an online ESP community. Interactive Learning Environments, 24(7), 1629-1646.

https://doi.org/10.1080/10494820.2015.1041402

Zimmerman, W. A., \& Kulikowich, J. M. (2016). Online learning self-efficacy in students with and without online learning Experience. American Journal of Distance Education, 30(3), 180-191.https://doi.org/10.1080/08923647.2 016.1193801 


\section{Anexo I}

\section{Escala de aprendizaje autogestivo}

El Grupo de investigación en Procesos Psicológicos y Sociales y el Laboratorio de Psicología Tecnología y Salud a través del proyecto PAPIME PE302819, te invitan a responder el siguiente cuestionario que tiene por objetivo conocer las situaciones a las que te enfrentas como estudiante en un sistema de educación a distancia. La información que proporciones será totalmente anónima y confidencial, solo se manejarán resultados para fines de la investigación.

De antemano, gracias por tu participación.

(Este formulario se creó en la Facultad de Estudios Superiores Iztacala, UNAM).

\section{*Obligatorio}

Accedo a participar de formar voluntaria y doy mi consentimiento para que la información que yo proporcione en este cuestionario sea utilizada para fines de investigación. *

$\mathrm{OSi}$

ONo

\section{DATOS DEMOGRÁFICOS}

\section{Sexo*}

O Hombre

O Mujer

\section{Edad*}

Tu respuesta

\section{Semestre*}

O Primero

O Segundo

O Tercero

O Cuarto

Q Quinto

O Sexto

O Séptimo

O Octavo

O Noveno

\section{Instrucciones}

A continuación, se presentarán una serie de palabras o frases en MAYÚSCULAS. Te pedimos que escribas 5 palabras independientes entre sí, que consideres se asocien con la palabra o frase.

Puedes utilizar verbos, adverbios, sustantivos, adjetivos u otros. Es muy importante solo usar palabras, procura no usar artículos ni preposiciones

Una vez que termines la lista enuméralas colocándolas en orden de importancia de acuerdo a lo que tú 
consideres que tiene, donde 1 corresponde a la más importante y 5 a la menos importante.

Recuerda que no hay respuestas correctas, ni incorrectas, por lo que deberás responder de acuerdo a lo que tu consideres relevante.

No pases a la siguiente sección sin haber terminado la numeración.

\section{Ejemplo}

Paso 1. Escribe 5 palabras relacionadas con MANZANA

Árbol, fruta, rica, roja, comida

Paso 2. Enumera las palabras anteriores en orden de importancia y entre paréntesis roja (4), fruta (1), árbol (3), rica (5), comida (2).

La información quedará registrada como en el ejemplo que se presenta a continuación:

\begin{tabular}{|c|c|}
\hline Paso 1 & MANZANA \\
\hline \multirow{10}{*}{$\begin{array}{l}\text { Puedes utilizar verbos, } \\
\text { adverbios, sustantivos, } \\
\text { adjetivos, entre otros. } \\
\text { Es muy importante que } \\
\text { sólo sean palabras } \\
\text { procura no usar } \\
\text { artículos, ni } \\
\text { preposiciones. }\end{array}$} & Palabra \\
\hline & árbol \\
\hline & Palabra \\
\hline & fruta \\
\hline & Palabra \\
\hline & rica \\
\hline & Palabra \\
\hline & roja \\
\hline & Palabra \\
\hline & Comida \\
\hline
\end{tabular}

<Atrás Continuar>

\begin{tabular}{|c|c|}
\hline Paso 2 & MANZANA \\
\hline Una vez que termines la & Palabra \\
\hline lista, el siguiente paso es & árbol (3) \\
\hline numerarlas en función de & Palabra \\
\hline la importancia o cercanía & fruta (1) \\
\hline que consideres tiene. & Palabra \\
\hline Donde 1 corresponde a la & rica (5) \\
\hline más cercana o más & Palabra \\
\hline importante y continúas con & roja (4) \\
\hline la numeración. & Palabra \\
\hline No pases a la siguiente & Comida (2) \\
\hline
\end{tabular}

hoja hasta que no hayas terminado.

Una vez completado el paso 2, puedes dar clic en continuar.

\section{APRENDER EN LÍNEA}

Paso 1. Escribe 5 palabras relacionadas

Paso 2. Enuméralas en orden de importancia

\section{Palabra:}

Tu respuesta Palabra:

\section{Palabra:}

Tu respuesta Palabra:

\section{Palabra:}

Tu respuesta Palabra:

\section{Palabra:}

Tu respuesta Palabra:

\section{Palabra:}

Tu respuesta Palabra:

\section{EXPERIENCIAS DE APRENDIZAJE EN LÍNEA}

Paso 1. Escribe 5 palabras relacionadas

Paso 2. Enuméralas en orden de importancia

\section{Palabra:}

Tu respuesta Palabra:

\section{Palabra:}

Tu respuesta Palabra:

\section{Palabra:}

Tu respuesta Palabra:

\section{Palabra:}

Tu respuesta Palabra:

\section{Palabra:}

Tu respuesta Palabra: 


\section{HABILIDADES DE UN ESTUDIANTE EN LÍNEA}

Paso 1. Escribe 5 palabras relacionadas

Paso 2. Enuméralas en orden de importancia

Palabra:

Tu respuesta Palabra:

\section{Palabra:}

Tu respuesta Palabra:

\section{Palabra:}

Tu respuesta Palabra:

\section{Palabra:}

Tu respuesta Palabra:

\section{Palabra:}

Tu respuesta Palabra:

\section{ESTRATEGIAS DE ESTUDIO EN} EDUCACIÓN EN LÍNEA

Paso 1. Escribe 5 palabras relacionadas Paso 2. Enuméralas en orden de importancia

\section{Palabra:}

Tu respuesta Palabra:

\section{Palabra:}

Tu respuesta Palabra:

\section{Palabra:}

Tu respuesta Palabra:

\section{Palabra:}

Tu respuesta Palabra:

\section{Palabra:}

Tu respuesta Palabra: 


\section{Anexo II}

\section{Escala de aprendizaje autogestivo}

El Grupo de investigación en Procesos Psicológicos y Sociales y el Laboratorio de Psicología Tecnología y Salud a través del proyecto PAPIME PE302819, te invitan a responder el siguiente cuestionario que tiene por objetivo conocer las situaciones a las que te enfrentas como estudiante en un sistema de educación a distancia. La información que proporciones será totalmente anónima y confidencial, solo se manejarán resultados para fines de la investigación.

De antemano, gracias por tu participación.

(Este formulario se creó en la Facultad de Estudios Superiores Iztacala, UNAM).

\section{*Obligatorio}

Accedo a participar de formar voluntaria y doy mi consentimiento para que la información que yo proporcione en este cuestionario sea utilizada para fines de investigación. *

$\mathrm{OSi}$

ONo

\section{DATOS DEMOGRÁFICOS}

1) Sexo*

O Hombre

O Mujer

2) Edad (años cumplidos)*

Tu respuesta

3) Estado civil*

O Soltero

O Casado(a)/Unión libre

$\bigcirc$ Divorciado

O Otro:

4) Ocupación (puedes marcar más de una opción)*

O Estudio

Trabajo

O Ama de casa

O Otro:

5) Si seleccionaste trabajo en la pregunta anterior ¿̨uántas horas por día trabajas? $(0,1,3,6$, 8...)
6) ¿Cuántas horas por día dedicas a tus estudios?

$(0,1,3,6,8 \ldots)$

Tu respuesta

7) ¿En qué semestre te encuentras?*

Tu respuesta

8) ¿Qué promedio tienes hasta el momento en tus estudios?

Tu respuesta

Instrucciones

A continuación, se presentan algunas afirmaciones que describen situaciones que el estudiante puede experimentar, lee cada una de ellas y marca la casilla que mejor describa la medida en que estás de acuerdo o en desacuerdo. Donde:

1 Totalmente en desacuerdo

2 En desacuerdo

$3 \mathrm{Ni}$ de acuerdo ni en desacuerdo

4 De acuerdo

5 Totalmente de acuerdo

No hay respuestas buenas ni malas, por lo que debes responder de la forma más veraz posible. 
1.Leer el material del curso forma parte de mi aprendizaje. *

$\begin{array}{llllllll} & 1 & 2 & 3 & 4 & 5 & \\ \text { Totalmente en desacuerdo } & 0 & 0 & 0 & \bigcirc & \bigcirc & \text { Totalmente de acuerdo }\end{array}$

2. Ser organizado/a es clave en mi éxito como estudiante. *

$\begin{array}{lllllll} & 1 & 2 & 3 & 4 & 5 & \\ \text { Totalmente en desacuerdo } & 0 & 0 & 0 & 0 & \bigcirc & \text { Totalmente de acuerdo }\end{array}$

3. Analizar la información me facilita el aprendizaje. *

$\begin{array}{lllllll} & 1 & 2 & 3 & 4 & 5 & \\ \text { Totalmente en desacuerdo } & 0 & 0 & 0 & 0 & \bigcirc & \text { Totalmente de acuerdo }\end{array}$

4. Mi autorregulación me permite mantener el objetivo académico. *

$\begin{array}{llllllll} & 1 & 2 & 3 & 4 & 5 & \\ \text { Totalmente en desacuerdo } & 0 & 0 & 0 & 0 & 0 & \text { Totalmente de acuerdo }\end{array}$

5. Me estreso al tener cercanas las fechas de entrega de las actividades. *

$\begin{array}{llllllll} & 1 & 2 & 3 & 4 & 5 & \\ \text { Totalmente en desacuerdo } & \bigcirc & \bigcirc & \bigcirc & \bigcirc & \bigcirc & \text { Totalmente de acuerdo }\end{array}$

6. Cuento con un equipo de cómputo adecuado para la realización de mis actividades

$\begin{array}{llllllll} & 1 & 2 & 3 & 4 & 5 & \\ \text { Totalmente en desacuerdo } & 0 & 0 & 0 & \bigcirc & \bigcirc & \text { Totalmente de acuerdo }\end{array}$

7. Me siento satisfecho con mis calificaciones. *

$\begin{array}{lllllll} & 1 & 2 & 3 & 4 & 5 & \\ \text { Totalmente en desacuerdo } & 0 & 0 & 0 & \bigcirc & \bigcirc & \text { Totalmente de acuerdo }\end{array}$

8. Tengo un manejo adecuado de la tecnología que apoya el desarrollo de mis actividades. *

$\begin{array}{llllllll} & 1 & 2 & 3 & 4 & 5 & \\ \text { Totalmente en desacuerdo } & \bigcirc & \bigcirc & \bigcirc & \bigcirc & \bigcirc & \text { Totalmente de acuerdo }\end{array}$

9. Me provoca ansiedad la falta de comunicación.

$\begin{array}{lllllll} & 1 & 2 & 3 & 4 & 5 & \\ \text { Totalmente en desacuerdo } & 0 & 0 & 0 & 0 & 0 & \text { Totalmente de acuerdo }\end{array}$

10. Cuando desconozco el tema de la actividad afecta mi estado de ánimo. *

$\begin{array}{lllllll} & 1 & 2 & 3 & 4 & 5 & \\ \text { Totalmente en desacuerdo } & 0 & 0 & 0 & 0 & 0 & \text { Totalmente de acuerdo }\end{array}$

11. Cuando las instrucciones de la actividad son difíciles afecta mi estado de ánimo. *

$\begin{array}{llllllll} & 1 & 2 & 3 & 4 & 5 & \\ \text { Totalmente en desacuerdo } & 0 & \bigcirc & \bigcirc & \bigcirc & \bigcirc & \text { Totalmente de acuerdo }\end{array}$

12. Mi nivel de autonomía fortalece mi proceso de aprendizaje. *

$\begin{array}{llllllll} & 1 & 2 & 3 & 4 & 5 & \\ \text { Totalmente en desacuerdo } & \bigcirc & \bigcirc & \bigcirc & \bigcirc & \bigcirc & \text { Totalmente de acuerdo }\end{array}$


13. Cuento con habilidades tecnológicas para estudiar en línea. *

$\begin{array}{llllllll} & 1 & 2 & 3 & 4 & 5 & \\ \text { Totalmente en desacuerdo } & \bigcirc & \bigcirc & \bigcirc & \bigcirc & \bigcirc & \text { Totalmente de acuerdo }\end{array}$

14. La innovación tecnológica hace de mi aprendizaje una meta interesante. *

$\begin{array}{llllllll} & 1 & 2 & 3 & 4 & 5 & \\ \text { Totalmente en desacuerdo } & 0 & 0 & 0 & \bigcirc & \bigcirc & \text { Totalmente de acuerdo }\end{array}$

15. Soy autodidacta para alcanzar los aprendizajes. *

$\begin{array}{lllllll} & 1 & 2 & 3 & 4 & 5 & \\ \text { Totalmente en desacuerdo } & \bigcirc & \bigcirc & \bigcirc & \bigcirc & \bigcirc & \text { Totalmente de acuerdo }\end{array}$

16. Soy responsable de mis estudios. *

$\begin{array}{lllllll} & 1 & 2 & 3 & 4 & 5 & \\ \text { Totalmente en desacuerdo } & \bigcirc & \bigcirc & \bigcirc & \bigcirc & \bigcirc & \text { Totalmente de acuerdo }\end{array}$

17. El tiempo que dedico como estudiante en línea me ayuda a desarrrollar habilidades tecnológicas para realizar mis actividades. *

$\begin{array}{llllllll} & 1 & 2 & 3 & 4 & 5 & \\ \text { Totalmente en desacuerdo } & \bigcirc & \bigcirc & 0 & \bigcirc & \bigcirc & \text { Totalmente de acuerdo }\end{array}$

18. El tiempo que dedico al estudio me lleva a adquirir habilidades. *

$\begin{array}{lllllll} & 1 & 2 & 3 & 4 & 5 & \\ \text { Totalmente en desacuerdo } & 0 & 0 & 0 & 0 & \bigcirc & \text { Totalmente de acuerdo }\end{array}$

19. El tiempo que dedico a la realización de mis actividades me permite desarrollar habilidades. *

$\begin{array}{llllllll} & 1 & 2 & 3 & 4 & 5 & \\ \text { Totalmente en desacuerdo } & \bigcirc & \bigcirc & \bigcirc & \bigcirc & \bigcirc & \text { Totalmente de acuerdo }\end{array}$

20. Mis habilidades de comunicación influyen en mi aprendizaje. *

$\begin{array}{lllllll} & 1 & 2 & 3 & 4 & 5 & \\ \text { Totalmente en desacuerdo } & \bigcirc & \bigcirc & \bigcirc & \bigcirc & \bigcirc & \text { Totalmente de acuerdo }\end{array}$

21. Mi creatividad es imprescindible en la realización de las actividades académicas. *

$\begin{array}{lllllll} & 1 & 2 & 3 & 4 & 5 & \\ \text { Totalmente en desacuerdo } & \bigcirc & 0 & \bigcirc & \bigcirc & \bigcirc & \text { Totalmente de acuerdo }\end{array}$

22. Adquiero conocimientos cuando realizo investigación bibliográfica.*

$\begin{array}{llllllll} & 1 & 2 & 3 & 4 & 5 & \\ \text { Totalmente en desacuerdo } & 0 & 0 & 0 & \bigcirc & \bigcirc & \text { Totalmente de acuerdo }\end{array}$

23. Soy autodidacta. *

$\begin{array}{llllllll} & 1 & 2 & 3 & 4 & 5 & \\ \text { Totalmente en desacuerdo } & 0 & \bigcirc & \bigcirc & \bigcirc & \bigcirc & \text { Totalmente de acuerdo }\end{array}$

24. El tiempo que dedico al estudio determina mis resultados en el aprendizaje. *

$\begin{array}{lllllll} & 1 & 2 & 3 & 4 & 5 & \\ \text { Totalmente en desacuerdo } & 0 & 0 & 0 & 0 & 0 & \text { Totalmente de acuerdo }\end{array}$


25. La comprensión de las lecturas es importante para mi. *

$\begin{array}{llllllll} & 1 & 2 & 3 & 4 & 5 & \\ \text { Totalmente en desacuerdo } & 0 & 0 & 0 & \bigcirc & \bigcirc & \text { Totalmente de acuerdo }\end{array}$

26.Realizo un esfuerzo adicional para estudiar en línea. *

$\begin{array}{llllllll} & 1 & 2 & 3 & 4 & 5 & \\ \text { Totalmente en desacuerdo } & 0 & \bigcirc & \bigcirc & \bigcirc & \bigcirc & \text { Totalmente de acuerdo }\end{array}$

27. Soy constante para concretar mi aprendizaje. *

$\begin{array}{lllllll} & 1 & 2 & 3 & 4 & 5 & \\ \text { Totalmente en desacuerdo } & 0 & 0 & 0 & \bigcirc & \bigcirc & \text { Totalmente de acuerdo }\end{array}$

28. Me comprometo para lograr un buen aprendizaje. *

$\begin{array}{llllllll} & 1 & 2 & 3 & 4 & 5 & \\ \text { Totalmente en desacuerdo } & 0 & 0 & 0 & \bigcirc & \bigcirc & \text { Totalmente de acuerdo }\end{array}$

29. Los tiempos de aprendizaje flexibles son una motivación para mi. *

$\begin{array}{llllllll} & 1 & 2 & 3 & 4 & 5 & \\ \text { Totalmente en desacuerdo } & \bigcirc & \bigcirc & \bigcirc & \bigcirc & \bigcirc & \text { Totalmente de acuerdo }\end{array}$

30. Es un reto para mi aprender en línea. *

$\begin{array}{llllllll} & 1 & 2 & 3 & 4 & 5 & \\ \text { Totalmente en desacuerdo } & \bigcirc & \bigcirc & \bigcirc & \bigcirc & \bigcirc & \text { Totalmente de acuerdo }\end{array}$

31. Soy autodidacta para lograr aprendizajes. *

$\begin{array}{lllllll} & 1 & 2 & 3 & 4 & 5 & \\ \text { Totalmente en desacuerdo } & \bigcirc & 0 & 0 & \bigcirc & \bigcirc & \text { Totalmente de acuerdo }\end{array}$

32. Cuando me siento incomprendido por parte del tutor me estreso. *

$\begin{array}{lllllll} & 1 & 2 & 3 & 4 & 5 & \\ \text { Totalmente en desacuerdo } & \bigcirc & 0 & \bigcirc & \bigcirc & \bigcirc & \text { Totalmente de acuerdo }\end{array}$

33. Me genera frustración las actividades de aprendizaje. *

$\begin{array}{llllllll} & 1 & 2 & 3 & 4 & 5 & \\ \text { Totalmente en desacuerdo } & 0 & 0 & \bigcirc & \bigcirc & \bigcirc & \text { Totalmente de acuerdo }\end{array}$

34. Soy perseverante en mis actividades académicas. *

$\begin{array}{llllllll} & 1 & 2 & 3 & 4 & 5 & \\ \text { Totalmente en desacuerdo } & 0 & 0 & 0 & 0 & 0 & \text { Totalmente de acuerdo }\end{array}$

35. La autonomía es una de mis estrategias de aprendizaje. *

$\begin{array}{llllllll} & 1 & 2 & 3 & 4 & 5 & \\ \text { Totalmente en desacuerdo } & 0 & 0 & 0 & \bigcirc & \bigcirc & \text { Totalmente de acuerdo }\end{array}$

36. Mi deseo de superación me lleva a buscar la excelencia académica. *

$\begin{array}{llllllll} & 1 & 2 & 3 & 4 & 5 & \\ \text { Totalmente en desacuerdo } & \bigcirc & \bigcirc & \bigcirc & \bigcirc & \bigcirc & \text { Totalmente de acuerdo }\end{array}$


37. Cuando me frustro se ve afectado mi aprendizaje. *

$\begin{array}{llllllll} & 1 & 2 & 3 & 4 & 5 & \\ \text { Totalmente en desacuerdo } & 0 & 0 & 0 & \bigcirc & \bigcirc & \text { Totalmente de acuerdo }\end{array}$

38. La realización de las tareas me proporcionan certeza en al aprendizaje.*

$\begin{array}{llllllll} & 1 & 2 & 3 & 4 & 5 & \\ \text { Totalmente en desacuerdo } & 0 & \bigcirc & \bigcirc & \bigcirc & \bigcirc & \text { Totalmente de acuerdo }\end{array}$

39. Mi cansancio afecta el aprendizaje que debo adquirir. *

$\begin{array}{llllllll} & 1 & 2 & 3 & 4 & 5 & \\ \text { Totalmente en desacuerdo } & 0 & 0 & 0 & 0 & 0 & \text { Totalmente de acuerdo }\end{array}$

40. Experimento estrés durante mi aprendizaje. *

$\begin{array}{llllllll} & 1 & 2 & 3 & 4 & 5 & \\ \text { Totalmente en desacuerdo } & 0 & 0 & 0 & \bigcirc & \bigcirc & \text { Totalmente de acuerdo }\end{array}$

41. La comprensión es una habilidad que poseo. *

$\begin{array}{llllllll} & 1 & 2 & 3 & 4 & 5 & \\ \text { Totalmente en desacuerdo } & \bigcirc & \bigcirc & \bigcirc & \bigcirc & \bigcirc & \text { Totalmente de acuerdo }\end{array}$

42. La disciplina forma parte de mis habilidades. *

$\begin{array}{llllllll} & 1 & 2 & 3 & 4 & 5 & \\ \text { Totalmente en desacuerdo } & \bigcirc & \bigcirc & \bigcirc & \bigcirc & \bigcirc & \text { Totalmente de acuerdo }\end{array}$

43. La innovación es una característica que poseo como estudiante. *

$\begin{array}{llllllll} & 1 & 2 & 3 & 4 & 5 & \\ \text { Totalmente en desacuerdo } & 0 & 0 & 0 & \bigcirc & \bigcirc & \text { Totalmente de acuerdo }\end{array}$

